\title{
The content of triterpene saponins and phenolic compounds in American ginseng hairy root extracts and their antioxidant and cytotoxic properties
}

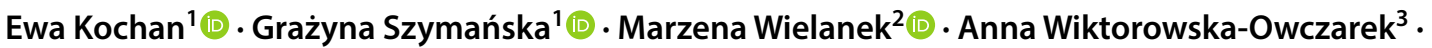 \\ Marta Jóźwiak-Bębenista ${ }^{3}$ (iD $\cdot$ Izabela Grzegorczyk-Karolak ${ }^{4}$ (D)
}

Received: 26 November 2018 / Accepted: 22 May 2019 / Published online: 29 May 2019

(C) The Author(s) 2019

\begin{abstract}
Panax quinquefolium is a perennial herb of the Araliaceae family native to North America. Its roots have been used in traditional and Chinese medicine. The aim of this study was to determine the phenolic profile of methanolic extracts of $P$. quinquefolium hairy roots cultivated in flasks and a bioreactor, as well as extracts from the roots of three-year-old field-grown plants. Additionally, the phenol and ginsenoside components of the tested extracts were identified by HPLC, and their antioxidant and cytotoxic properties were evaluated. The antioxidant effect was evaluated by FRAP (ferric reducing antioxidant power), and ABTS ([2,2'-azino-bis(3-ethylbenzothiazoline-6-sulfonic acid) cation scavenging tests, and their effect on the viability of the glioblastoma cell (T98G) line was measured using the 3-(4,5-dimethylthazol-2-yl)-2,5-diphenyltetrazolium bromide (MTT) assay. The LC-MS/MS analysis revealed the presence of 16 phenolic compounds identified as phenolic acids (ten compounds) or flavonoids (six compounds). The highest phenol content was observed in the transformed roots of flask-grown P. quinquefolium (1.6 $\mathrm{mg} \mathrm{g}^{-1}$ d.w.), followed by these grown in the bioreactor $\left(1.1 \mathrm{mg} \mathrm{g}^{-1}\right.$ d.w.). However, the highest ginsenoside content was found in the roots of the naturally-cultivated plants $\left(67.6 \mathrm{mg} \mathrm{g}^{-1} \mathrm{~d}\right.$.w. $)$. The methanolic extracts from hairy root culture of $P$. quinquefolium appear to have significant antioxidant and cytotoxic potential. Such transformed American ginseng root cultures could represent a potential source of bioactive metabolites for the food or pharmaceutical industry.
\end{abstract}

\section{Key message}

First time phenolic compound level, antioxidant and cytotoxic properties were estimated in hairy root cultures of Panax quinquefolium cultivated in flasks and nutrient sprinkle bioreactor. Ginsenosides content was also determined.

Keywords Ginsenosides $\cdot$ Phenolic compounds $\cdot$ Antioxidant activity $\cdot$ Hairy root cultures $\cdot$ Nutrient sprinkle bioreactor

\section{Introduction}

Communicated by Sergio J. Ochatt.

Electronic supplementary material The online version of this article (https://doi.org/10.1007/s11240-019-01633-3) contains supplementary material, which is available to authorized users.

Ewa Kochan

ewa.kochan@umed.lodz.pl

Extended author information available on the last page of the article
Antioxidants are chemical compounds that neutralize free radicals, which are formed physiologically in the organism from oxygen. They have many important functions: under normal physiological conditions, reactive oxygen species (ROS) act as mediators and regulators of plant metabolism. They affect the signaling between cells and within cells, regulate gene expression, and can serve as secondary relays in the processes of growth and cell death (Sukweenadhi et al. 2017). However, excessive production of ROS and exhaustion by the cell of its oxidative reserves, that is oxidative stress, results in the oxidation and structural modification 
of proteins, and the consequent disruption of their function (Van Breusegem and Dat 2006). Moreover, high ROS concentrations trigger and intensify the processes of biomolecule damage that may cause enzyme inactivation, DNA strand breaks and mutations, leading to the development of tumors (Kasote et al. 2015). At both the cellular and organism levels, antioxidant protection is provided by numerous enzymes and endogenous small molecular weight antioxidants such as ascorbic acid, uric acid, glutathione and tocopherols, as well as various other metabolites isolated from plants (Matkowski 2008; Kasote et al. 2015; Sukweenadhi et al. 2017).

One major group of plant antioxidants is constituted by phenolic compounds, which are involved in a number of physiological mechanisms, including antioxidant protection. Plant phenolics are mainly classified into five major groups: phenolic acids, flavonoids, lignans, stilbenes and tannins. These compounds possess an aromatic ring bearing one or more hydroxyl groups, and their structures may range from that of a simple phenolic molecule to that of a complex high-molecular weight polymer. The antioxidant activity of phenolic compounds depends on the structure, in particular the number and positions of the hydroxyl groups and the nature of the substitutions on the aromatic rings (Kasote et al. 2015).

Meanwhile, other groups of secondary metabolites have also been found to have antioxidant properties (Matkowski 2008; Jang et al. 2016). For example, it was revealed that some ginsenosides from $P$. ginseng protect rabbit pulmonary endothelium tissue from ROS toxicity (Lee et al. 2014). Ginsenosides are triterpene saponins isolated from different species of Panax such as $P$. ginseng, $P$. quinquefolium and P. notoginseng (Sun et al. 2011; Brown et al. 2013; Kang and Kim 2016). Nowadays more than 150 types of ginsenosides are known; they are subdivided into two main classes according to their sapogenin (terpenoid backbone): $\mathrm{Rb}$ and $\mathrm{Rg}$ groups. $\mathrm{Rb}$ ginsenosides, such as $\mathrm{Rb} 1, \mathrm{Rb} 2, \mathrm{Rc}$, $\mathrm{Rd}$ metabolites, are (20) $\mathrm{S}$ protopanaxadiol derivatives, while the $\operatorname{Rg}$ group, such as $\operatorname{Rg} 1$ and Re metabolites, have (20) S protopanaxatriol as a sapogenin (Biswas et al. 2017). Many pharmacological investigations (Christensen 2009; Tung et al. 2012; Lee and Kim 2014; Lee et al. 2014) indicate that ginsenosides are responsible for the adaptogenic, antidiabetic, antitumor (Wang and Zhang 2012; Pawar and Shivakumar 2012; Wang et al. 2015), anti-obesity (Marrelli et al. 2016) and neuroprotective (Radad et al. 2011) properties of ginseng.

Our previous research describes the successful acquisition of $P$. qiunquefolium (American ginseng) hairy root cultures and the optimization of ginsenoside production in this culture, both at small scale and when scaling up. Gamborg medium (B-5 medium) containing $30 \mathrm{~g} \mathrm{~L}^{-1}$ saccharose was the most favorable substrate for saponin accumulation
(Kochan et al. 2014). The study also evaluated the effect of different concentrations of $\mathrm{PO}_{4}{ }^{3-}, \mathrm{NH}_{4}{ }^{+}$and $\mathrm{NO}_{3}{ }^{-}$ions in the medium on ginsenoside production in $P$. quinquefolium hairy root culture: saponin yield was optimized by limiting the phosphate ion concentration to $0.83 \mathrm{mM}$ and reducing $\mathrm{NH}_{4}{ }^{+}$and $\mathrm{NO}_{3}{ }^{-}$ion concentrations to half their amounts in standard B-5 medium, (Kochan et al. 2016). In addition, after some modifications in medium composition, the levels of the studied ginsenosides were significantly higher in the nutrient sprinkle bioreactor than in control medium, and higher than in roots cultivated in flasks under optimal conditions (Kochan et al. 2016).

This work is a continuation of our research on the chemical composition of hairy roots of $P$. quinquefolium and serves to address the gap in current literature regarding the content of phenolic compounds in these cultures. Its aims were therefore to perform a qualitative and quantitative determination of the phenolic compound level in transformed $P$. quinquefolium roots, and to determine the levels of the predominant ginsenosides in a 10-year-old root culture.

The next step of our investigation was to evaluate the biological potential of the extracts of $P$. quinquefolium hairy roots, and determine the antioxidant properties of the extracts obtained from the transformed root cultures, both cultivated in shake flasks and in the nutrient sprinkle bioreactor, as well as their cytotoxic potential against a glioblastoma cell line (T98G). Furthermore, the in vitro findings were compared with those obtained for roots of field-cultivated plants.

\section{Materials and methods}

\section{Hairy root cultures of $P$. quinquefolium}

Agrobacterium rhizogenes ATCC 15834 was used to obtain hairy root cultures of $P$. quinquefolium. The transformation process and its and confirmation were described in detail in an earlier paper (Kochan et al. 2012). The culture was grown in hormone-free, modified B-5 medium containing $30 \mathrm{~g} \mathrm{~L}^{-1}$ sucrose: the medium was a standard B-5 medium but with half the concentrations of $\mathrm{NH}_{4}{ }^{+}$and $\mathrm{NO}_{3}{ }^{-}$ions and a phosphate ion content restricted to $0.83 \mathrm{mM}$. The roots were incubated in $300 \mathrm{~mL}$ shake Erlenmeyer flasks placed on a rotary shaker $(100 \mathrm{rpm})$ in darkness at $26 \pm 2{ }^{\circ} \mathrm{C}$. The culture was maintained by regular subcultures every 28 days. The average inoculum size was about $320 \mathrm{mg}$ fresh weight (f.w.) and $29.8 \mathrm{mg}$ dry weight (d.w.) per flask. The hairy roots was also cultured in scale up using a nutrient sprinkle bioreactor (Supplementary Fig. 1). The bioreactor type and culturing conditions were as described earlier (Kochan et al. 
2012). The bioreactor was inoculated using roots from five flasks, of approx. $24.5 \mathrm{~g}$ fresh weight and $2.19 \mathrm{~g}$ dry weight.

\section{Root form field cultivation}

For comparison, the roots of 3-year-old field-grown plants (FR) were also tested. These were obtained from the Academy of Agriculture in Lublin, Poland.

\section{Sample preparation}

The roots from field cultivation and after 28 days of growth in in vitro conditions, were dried at room temperature and subjected to extraction in $80 \%$ methanol and solid phase extraction as described earlier (Kochan et al. 2017). The dried hydromethanolic extracts, taken from the three types of cultures, viz. hairy roots cultured in shake flasks, nutrient sprinkle bioreactor cultures and roots from field plants, were weighed and used for HPLC analysis of ginsenosides and phenolic compounds.

\section{HPLC analysis}

The samples were tested for the presence of six ginsenosides (Rb1, Rb2, Rc, Rd, Re, Rg1 purchased from C. Roth $\mathrm{GmbH}+\mathrm{Co}$ Karlsruhe, Germany) using a liquid chromatography system consisting of an Agilent Technology 1200 apparatus, a ZORBAX Eclipse XDB-C18 $(150 \times 4.6 \mathrm{~mm}$, $5 \mu \mathrm{m})$ column, Quat Pump and UV-Vis DAD type detector, and an Agilent Technology set combined with Agilent ChemStation 2001-2010 software. The other details of this analysis are described in an earlier report (Kochan et al. 2017). The ginsenoside content was expressed as mg per $\mathrm{g}$ of dry weight.

Chromatographic analysis of the phenolic compounds was carried out using an HPLC system (Dionex, Sunnyvale, USA) equipped with a photodiode-array detector. The compounds were separated on an RP column (aQ Hypersil GOLD, $250 \times 4.6 \mathrm{~mm}, 5 \mu \mathrm{m}$ ) coupled with a guard column (GOLD aQ Drop-In guards, $10 \times 4 \mathrm{~mm}, 5 \mu \mathrm{m}$, Polygen, Gliwice, Poland) at $25{ }^{\circ} \mathrm{C}$ using a mobile phase composed of water (A) and methanol (B), both with $0.1 \%$ formic acid. The linear gradient was started after 2 min of isocratic elution with 5\% B and was slowly increased over $30 \mathrm{~min}$ to $55 \%$ $\mathrm{B}$, followed by $5 \mathrm{~min}$ of isocratic elution, increasing to $70 \%$ within $10 \mathrm{~min}$. After an isocratic step with $70 \%$ B for $5 \mathrm{~min}$, the gradient was returned to the initial $5 \% \mathrm{~B}$ within 2 min to re-equilibrate the column for the next $3 \mathrm{~min}$. The flow rate was $1 \mathrm{~mL} \mathrm{~min}^{-1}$, and the absorbance was measured at 210 , $235,280,325$ and $375 \mathrm{~nm}$.

LC-MS/MS of phenolic compounds was carried out using an API LC/MS/MS system (Applera, USA) with electrospray ionization (ESI) source equipped with a Dionex
(Germany) HPLC system. Separation was achieved on an aQ Hypersil GOLD column $(\mathrm{C} 18,2.1 \mathrm{~mm} \times 150 \mathrm{~mm}, 5 \mu \mathrm{m})$ at $30{ }^{\circ} \mathrm{C}$ using a gradient as described above for HPLC and a flow rate of $0.2 \mathrm{~mL} \mathrm{~min}^{-1}$. Detection was performed in the negative ion mode with the following conditions: drying gas $\left(\mathrm{N}_{2}\right) 11.0 \mathrm{~L} \mathrm{~min}^{-1}$, temperature $350{ }^{\circ} \mathrm{C}$, nitrogen nebulizer pressure $40 \mathrm{psi}$, capillary voltage $4.5 \mathrm{~kW}$, a detector gain of $1600 \mathrm{~V}$, fragmentation voltage $100 \mathrm{~V}$ and full scan range from 100 to $900 \mathrm{~m} / \mathrm{z}$.

The phenolic compounds present in the hairy root extracts were identified by comparing the retention times, on-line UV absorption spectra and MS spectra of the analyzed samples with respective data obtained from the analysis of reference standards (all purchased from Sigma Aldrich). Quantification was based on the calibration curve for standards constructed over the range of 5-200 $\mu \mathrm{g} \mathrm{mL}^{-1}$, the linearity of the calibration curve was verified by the correlation coefficient $\left(r^{2}=0.9994\right)$. The content phenolic compounds was expressed as $\mu \mathrm{g}$ per $\mathrm{g}$ of dry weight.

\section{Total phenolic determination}

The total phenolic content (TPC) was determined using Folin-Ciocalteu reagent as described previously (Kochan et al. 2015). The absorbance of the resulting blue color was measured at $765 \mathrm{~nm}$ on a Rayleigh UV-1601 spectrophotometer (Beijing Reyleigh Corp. China) versus a blank sample. TPC were expressed as gallic acid mg equivalents (GAE) per gram of dry extract. The results are presented as means of triplicate analyses.

\section{Determination of antioxidant activities}

The antioxidant potential of the methanolic extracts from the hairy root cultures and the field roots of $P$. quinquefolium was evaluated using two in vitro assays: ABTS $\left(2,2^{\prime}\right.$-azinobis-(3ethylbenzothiazoline-6-sulfonic acid) cation and ferric reducing antioxidant power (FRAP). Sample preparation and analysis was performed as described previously (Kochan et al. 2015). The results of the ABTS analysis were expressed as $\mathrm{EC}_{50}\left(\mu \mathrm{g} \mathrm{mL}^{-1}\right)$ : the concentration of the sample at which $50 \%$ maximum scavenging activity was recorded. The antioxidant potential of the sample, measured by the FRAP method, was determined against a standard $\mathrm{FeSO}_{4} \times 7 \mathrm{H}_{2} \mathrm{O}$ value calculated from a calibration curve plotted with concentrations from 0 to $2000 \mu \mathrm{M}$.

\section{Determination of cytotoxic properties}

\section{Cell cultures}

The T98G glioma cell line was purchased from the American Type Culture Collection (ATCC; Rockville, MD, USA). 
The cells were cultured in 25-mL flasks in medium composed of Advanced MEM supplemented with 10\% fetal bovine serum, $2 \mathrm{mM}$ glutamine and a penicillin-streptomycin solution, in a humidified atmosphere of $95 \%$ air and 5\% $\mathrm{CO}_{2}$ at $37{ }^{\circ} \mathrm{C}$. For subcultures, cells were harvested every third day in trypsin-EDTA ( $0.25 \%$ trypsin, 1 mM EDTA) solution.

\section{MTT assay}

The viability of the glioblastoma cell line (T98G) was measured using the 3-(4,5-dimethylthazol-2-yl)-2,5-diphenyltetrazolium bromide (MTT; Sigma-Aldrich Chemical Co. Ltd; St. Louis, USA) conversion method. Cells were seeded (50 000 cells/well) into 96 -well plates. The treated cells were incubated for 24 and $48 \mathrm{~h}$ with extracts isolated from hairy root cultures and without tested extract (control group). After incubation, $50 \mu \mathrm{l} \mathrm{MTT}\left(1 \mathrm{mg} \mathrm{mL}^{-1}\right.$, Sigma) was added and the plates were incubated at $37{ }^{\circ} \mathrm{C}$ for $3 \mathrm{~h}$. At the end of the experiment, the cells were exposed to $100 \mu \mathrm{l}$ dimethyl sulphoxide, which enabled the release of the blue reaction product: formazan. The absorbance at $570 \mathrm{~nm}$ was read on a microplate reader and results were expressed as a percentage of the absorbance measured in control cells.

\section{Statistical analysis}

All treatments were performed in triplicate. Data was analysed using the Kruskal-Wallis test. Any relationships were considered significant at $p \leq 0.05$. Statistica Version 13.1 software was used for all statistical analyses (STATSoft).

\section{Results}

\section{Ginsenoside production}

The ginsenoside content was determined in three sets of plants: hairy root cultures cultivated in shake flasks (FHR), cultures grown in the nutrient sprinkle bioreactor (BHR), and the roots of three-year-old $P$. quinquefolium plants cultivated in the field (FR). The obtained results indicated significant differences in saponin levels between the flask and bioreactor cultures, but also between the roots produced in vitro and those grown in vivo (Table 1). After 28-day cultivation, the roots growing in the bioreactor proved to be a better source of all ginsenosides than the cultures cultivated in shake flasks: The bioreactor cultures accumulated $35.1 \pm 5.25 \mathrm{mg} \mathrm{g}^{-1}$ d.w. of total saponins (TGC), this being 2.8 times more than in the flask-grown roots $\left(12.5 \pm 3.02 \mathrm{mg} \mathrm{g}^{-1} \mathrm{~d}\right.$.w. $)$. The ginsenoside accumulation in roots of natural grown plants was $67.6 \pm 3.19 \mathrm{mg} \mathrm{g}^{-1}$ d.w.
Table 1 Ginsenoside accumulation in hairy root culture (cultivated in bioreactor (BHR) and shake flasks (FHR)) and roots of field (FR) grown $P$. quinquefolium plants

\begin{tabular}{lrrr}
\hline Ginsenoside & \multicolumn{3}{c}{ Ginsenoside content $\left[\mathrm{mg} \mathrm{g}^{-1}\right.$ d.w. $]$} \\
\cline { 2 - 4 } & \multicolumn{1}{l}{ FHR } & \multicolumn{1}{c}{ BHR } & \multicolumn{1}{l}{ FR } \\
\hline Rb1 & $3.2^{\mathrm{a}} \pm 0.45$ & $9.5^{\mathrm{b}} \pm 0.083$ & $30.22^{\mathrm{c}} \pm 4.51$ \\
Rb2 & $0.9^{\mathrm{a}} \pm 0.12$ & $2.5^{\mathrm{b}} \pm 0.65$ & $0.37^{\mathrm{c}} \pm 0.12$ \\
Rc & $1.8^{\mathrm{a}} \pm 0.11$ & $5.1^{\mathrm{b}} \pm 0.46$ & $7.94^{\mathrm{c}} \pm 1.05$ \\
Rd & $2.2^{\mathrm{a}} \pm 0.76$ & $6.3^{\mathrm{b}} \pm 0.75$ & $3.44^{\mathrm{a}} \pm 0.78$ \\
Total Rb group & $8.1^{\mathrm{a}} \pm 0.48$ & $23.4^{\mathrm{b}} \pm 3.92$ & $41.97^{\mathrm{c}} \pm 7.51$ \\
Re & $1.4^{\mathrm{a}} \pm 0.49$ & $4.0^{\mathrm{b}} \pm 0.26$ & $23.30^{\mathrm{c}} \pm 1.95$ \\
Rg1 & $3.0^{\mathrm{a}} \pm 0.61$ & $7.7^{\mathrm{b}} \pm 0.47$ & $2.33^{\mathrm{a}} \pm 0.13$ \\
Total Rg group & $4.4^{\mathrm{a}} \pm 0.52$ & $11.7^{\mathrm{b}} \pm 1.05$ & $25.63^{\mathrm{c}} \pm 4.12$ \\
Total content & $12.5^{\mathrm{a}} \pm 3.02$ & $35.1^{\mathrm{b}} \pm 5.25$ & $67.6^{\mathrm{c}} \pm 3.19$ \\
\hline
\end{tabular}

The values were mean \pm standard error (SE). The different letters in rows mean statistically significant differences indicated using Kruskal-Wallis test

All root cultures accumulated more $\mathrm{Rb}$ group saponins (calculated as the sum of Rb1, Rb2, Rc and Rd) than those of the $\mathrm{Rg}$ group (calculated as the sum of $\mathrm{Rg} 1$ and $\mathrm{Re}$ ). Although the $\mathrm{Rb}$ derivatives accounted for more than $60 \%$ of all tested saponin compounds in all analyzed plant materials, the ginsenoside content varied, both individually and as a percentage of total saponin content (Table 1, Supplementary Fig. 2). Although the levels of particular compounds in the hairy roots varied depending on the culture conditions, the proportions of individual compounds with regard to total saponin production were similar in both in vitro cultures. However, significant differences were observed between the roots of natural grown $P$. quinquefolium and the in vitro cultures with regard to the levels of the secondary compounds and their percentage share of the total content of saponins.

Of the $\mathrm{Rb}$ group, ginsenoside $\mathrm{Rb} 1$ predominated in all investigated samples; however, its amount differed between in vitro and in vivo grown plants $\left(30.2 \pm 4.51 \mathrm{mg} \mathrm{g}^{-1}\right.$ d.w. for FR, $9.5 \pm 0.083 \mathrm{mg} \mathrm{g}^{-1}$ d.w. for BHR, and $3.2 \pm 0.45 \mathrm{mg} \mathrm{g}^{-1}$ d.w. for FHR). Rb1 constituted around $45 \%$ of total ginsenoside content for FR, but only $25 \%$ for each HR (hairy root) culture (Supplementary Fig. 2). The level of Rb2 was significantly higher in the HR cultures: the bioreactor and the flask cultures produced respectively 6.7- and 2.4-times more $\mathrm{Rb} 2$ than the FR cultures. $\mathrm{Rb} 2$ concentration represented 7\% of total ginsenoside production in HR, but only $0.5 \%$ in FR.

The BHR plants demonstrated the highest Rd content. Following this, Rd constituted $17 \%$ of the total ginsenoside content in HR, independent of the cultivation conditions, but only $5 \%$ of saponin production in the field-grown plants (Supplementary Fig. 2). The predominant protopanaxatriol derivatives were $\mathrm{Rg} 1$ in the HR cultures, and $\mathrm{Re}$ in the FR roots (Table 1, Supplementary Fig. 2). 


\section{Phenolic compound production}

Negative ionization LC-ESI-MS/MS was used to analyze the structures of the hydromethanolic extracts of $P$. quinquefolium roots. The identification was based on a comparison of their retention times, UV absorption spectra and mass data with authentic standards. Sixteen phenolic compounds were found: ten phenolic acids and six flavonoids (Supplementary Table 1, Supplementary Fig. 3). Although all analyzed samples showed a similar profile, the individual compounds were found in different proportions depending on the plant material (Table 2).

As with the ginsenosides, the polyphenol content depended not only on the type of plant material (i.e. hairy roots or roots from field-grown plants), but also on the cultivation conditions (flasks or bioreactor). The greatest content of phenolic compounds was found in the flask cultures $\left(1588.7 \pm 102.5 \mu \mathrm{g} \mathrm{g}^{-1}\right.$ d.w.) (Table 2), being 1.5-times higher than in the other analyzed extracts. The levels of individual metabolites were highly diverse and ranged from trace amounts to $840.8 \pm 41.6 \mu \mathrm{g} \mathrm{g}^{-1}$ d.w. The predominant compound of both the FHR and BHR plants was chlorogenic acid; however, its level was twice as high in the FHR cultures, and accounted for almost $40 \%$ of all phenols in FHR and more than 50\% in BHR. The main constituent in the root extracts of field-grown plants was $\alpha$-resorcylic acid $\left(323.0 \pm 17.7 \mu \mathrm{g} \mathrm{g}^{-1} \mathrm{~d} . \mathrm{w}\right)$, which was also found in similar levels in the in vitro cultivated roots.
Flavonoids were found to be present only in small concentrations, with catechin being the predominant metabolite of this type $\left(30.7 \pm 1.4-60.7 \pm 2.0 \mu \mathrm{g} \mathrm{g}^{-1}\right.$ d.w. depending on the sample). These metabolites only constituted a relevant percentage of identified phenolic compounds-in the BHR culture (approximately 9\%) and only 3.6\% and $6.2 \%$ in the FHR and FR cultures, respectively.

Total polyphenol content (TPC) was also investigated using the Folin-Ciocalteu method. TPC level was found to be higher in the HR cultures than the FR plants. The highest TPC was observed in the shake flask cultures $\left(17.7 \pm 0.67 \mathrm{mg} \mathrm{GAE} \mathrm{g}^{-1}\right.$ of dry extract); this level was about 20\% higher than the BPH cultures. The TPC level was five-times higher in the flask cultures than in the FR roots.

Total ginsenoside content (TGC) was found to be inversely related to TPC in all three sets of samples. A strong negative correlation was identified between the two tested parameters (Pearson correlation coefficient: $r=0.9899)$.

\section{Antioxidant potential}

The antioxidant activity of the FHR and BHR extracts was compared with that of the roots of the field-grown plants using the FRAP and ABTS assays (Table 3). All studied extracts showed antioxidant potential. The BHR extracts were characterized by both higher FRAP reduction ability
Table 2 Identified phenolic compound accumulation in hairy root culture (cultivated in bioreactor (BHR) and shake flasks (FHR)) and roots of field (FR) grown $P$. quinquefolium plants

\begin{tabular}{|c|c|c|c|}
\hline \multirow[t]{2}{*}{ Compounds } & \multicolumn{3}{|c|}{ Content $\left[\mu \mathrm{g} \mathrm{g}^{-1}\right.$ d.w. $]$} \\
\hline & FHR & BHR & FR \\
\hline$\alpha$-Resorcylic acid (3,5-dhBA) & $320.96^{\mathrm{a}} \pm 6.2$ & $314.8^{\mathrm{a}} \pm 14.7$ & $323.0^{\mathrm{a}} \pm 17.7$ \\
\hline Vanillic acid & $9.8^{\mathrm{a}} \pm 0.7$ & $12.3^{\mathrm{a}} \pm 1.1$ & $24.9^{\mathrm{b}} \pm 1.5$ \\
\hline Syringic acid & $310.8^{\mathrm{a}} \pm 1.9$ & $47.2^{\mathrm{b}} \pm 9.0$ & $160.9^{\mathrm{c}} \pm 8.9$ \\
\hline Ferulic acid & - & $11.8^{\mathrm{a}} \pm 0.9$ & $35.3^{\mathrm{b}} \pm 2.0$ \\
\hline$(+)$-Catechin & $30.7^{\mathrm{a}} \pm 1.4$ & $60.7^{\mathrm{b}} \pm 2.0$ & $55.8^{\mathrm{b}} \pm 5.1$ \\
\hline 4-Hydroxybenzoic acid & $28.6^{\mathrm{a}} \pm 0.8$ & $15.4^{\mathrm{b}} \pm 1.1$ & $45.2^{\mathrm{c}} \pm 2.9$ \\
\hline Ellagic acid & $29.5^{\mathrm{a}} \pm 1.7$ & $49.6^{\mathrm{b}} \pm 0.1$ & $14.3^{\mathrm{c}} \pm 3.1$ \\
\hline Hesperidin & Trace & $10.6 \pm 2.3$ & Trace \\
\hline Gentisic acid (2,5-dhBA) & $233.0^{\mathrm{a}} \pm 13.2$ & $92.0^{\mathrm{b}} \pm 6.6$ & $260.1^{\mathrm{a}} \pm 26.5$ \\
\hline Chlorogenic acid & $840.8^{a} \pm 41.6$ & $422.1^{\mathrm{b}} \pm 6.4$ & $61.6^{c} \pm 13.4$ \\
\hline Caffeic acid & $12.1^{\mathrm{a}} \pm 2.5$ & $9.7^{\mathrm{a}} \pm 1.1$ & $2.0^{\mathrm{b}} \pm 0.7$ \\
\hline$p$-Coumaric acid & $16.6^{\mathrm{a}} \pm 2.2$ & $31.5^{\mathrm{b}} \pm 2.2$ & $14.5^{\mathrm{a}} \pm 1.5$ \\
\hline Luteolin & $2.6^{\mathrm{a}} \pm 0.4$ & $8.8^{\mathrm{b}} \pm 0.7$ & Trace \\
\hline Myricetin & $14.4^{\mathrm{a}} \pm 3.7$ & $11.8^{\mathrm{a}} \pm 2.6$ & $6.8^{\mathrm{b}} \pm 0.7$ \\
\hline Rutin & $1.9^{\mathrm{a}} \pm 0.5$ & $6.4^{\mathrm{b}} \pm 0.5$ & Trace \\
\hline Quercetin & Trace & Trace & Trace \\
\hline Total & $1588.7^{\mathrm{a}} \pm 102.5$ & $1104.7^{\mathrm{b}} \pm 74.5$ & $1005.5^{\mathrm{b}} \pm 63.2$ \\
\hline
\end{tabular}

The values were mean \pm standard error (SE). The different letters in rows mean statistically significant differences indicated using Kruskal-Wallis test 
Table 3 Antioxidant and cytotoxic activity of methanolic extracts of hairy root culture (cultivated in bioreactor (BHR) and shake flasks (FHR)) and roots of field (FR) grown $P$. quinquefolium plants

\begin{tabular}{|c|c|c|c|c|}
\hline \multirow[t]{2}{*}{ Plant material } & \multicolumn{2}{|c|}{ Antioxidant assay } & \multicolumn{2}{|c|}{ Cytotoxic activity } \\
\hline & FRAP $^{\mathrm{i}}$ & $\mathrm{ABTS}^{\mathrm{ii}}$ & $\mathrm{IC}_{50}$ after $24 \mathrm{~h}$ & $\mathrm{IC}_{50}$ after $72 \mathrm{~h}$ \\
\hline BHR & $112.99 \pm 1.25^{\mathrm{a}}$ & $177.99 \pm 3.16^{\mathrm{c}}$ & $0.301^{\mathrm{a}} \pm 0.051$ & $0.234^{\mathrm{a}} \pm 0.032$ \\
\hline FHR & $94.14 \pm 3.05^{\mathrm{b}}$ & $228.60 \pm 4.28^{\mathrm{b}}$ & $0.313^{\mathrm{a}} \pm 0.034$ & $0.268^{\mathrm{a}} \pm 0.05$ \\
\hline FR & $58.82 \pm 2.53^{\mathrm{c}}$ & $320.97 \pm 1.42^{\mathrm{c}}$ & $1.179^{\mathrm{b}} \pm 0.22$ & $0.683^{\mathrm{b}} \pm 0.07$ \\
\hline
\end{tabular}

${ }^{\mathrm{i}}$ Ferric reducing antioxidant power expressed in $\mu \mathrm{mol} \mathrm{Fe}(\mathrm{II}) \mathrm{g}^{-1}$ extract d.w., 15 min of incubation

${ }^{i i}$ Radical scavenging activity was determined using ABTS method and was expressed as $\mathrm{EC}_{50}$ meaning the concentration of sample $\left[\mathrm{mg} \mathrm{mL}^{-1}\right]$ showing $50 \%$ of maximal radical scavenging activity, 10 min of incubation

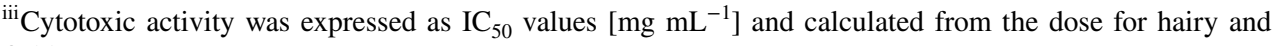
field root extracts

The values are the mean \pm SE. The means in column with the same letter do not differ statistically according to the Kruskall-Wallis test $(p \leq 0.05)$ $\left(112.99 \pm 1.25 \mu \mathrm{mol} \mathrm{Fe}(\mathrm{II}) \mathrm{g}^{-1}\right.$ d.w. of extract) and ABTS radical scavenging $\left(177.99 \pm 3.16 \mu \mathrm{g} \mathrm{m}^{-1}\right)$ than the other types of extract: the FHR extracts demonstrated $20 \%$ lower activity, and the FR extracts about $50 \%$.

\section{The effects of FHR and BHR on the viability of glioblastoma cell line (T98G)}

In the present studies, the cytotoxicity of tested extracts was determined using glioblastoma cell line T98G. Cell viability was determined against extracts in the concentration range 0.02 to $0.8 \mathrm{mg} \mathrm{mL}^{-1}$. The obtained results are presented in Fig. 1. In general, a dose-dependent decrease in the survival of the studied cell line was observed, and 72-h incubation with extracts exerted stronger cytotoxic effects towards cells than 24-hour incubation. The highest cytotoxic potential was found for BHR $\left(\mathrm{IC}_{50}\right.$ value was $0.301 \pm 0.051 \mathrm{mg} \mathrm{mL}^{-1}$ after $24 \mathrm{~h}$, and $0.234 \pm 0.032 \mathrm{mg} \mathrm{mL}^{-1}$ after $72 \mathrm{~h}$ ) The extract from the in vivo grown plant showed only around $33 \%$ of this effectiveness.

\section{Discussion}

\section{Ginsenoside and phenolic compound production}

A greater total ginsenoside content was found in the FR culture than the in vitro HR cultures. However, to obtain such ginsenoside-rich material in natural cultivation conditions, a minimum of 3 years is necessary, and harvesting the root completely liquidates the crop. In contrast, hairy root cultures only require 28 days. Additionally transformed root cultures of $P$. quinquefolium possess higher or comparable ginseng saponin levels than roots from field-cultivated plants (Shi et al. 2007; Kim et al. 2014a, b; Kochan et al. 2016). There are also studies indicating that transformed root cultures can accumulate much larger amounts of biologically active metabolites than plants grown in vivo (Bathoju et al. 2017).

The FHR hairy root culture of $P$. quinquefolium in the present study produced 10 times more saponin than previously-tested adventitious $P$. ginseng roots, and twice that of transformed roots (Inomata et al. 1993; Mallol et al. 2001; Wang et al. 2012). In addition, these cultures synthesized more protopanaxadiol derivatives than protopanaxatriol ones, similarly to hairy roots of $P$. vietnamensis (Ha et al. 2016).

Our findings revealed 2.8-fold greater ginsenoside production in the nutrient sprinkle bioreactor culture than the flask culture. Also, research on P. ginseng and $P$. vietnamensis adventitious root culture indicates that ginsenoside production may be more efficient in bioreactor system than in small-scale culture (Wang et al. 2012; Linh et al. 2019). The differences in metabolite content observed between the flask and bioreactor cultures could be attributed to differences in nutrient supply, gas phase composition or concentration of gaseous components (oxygen, carbon dioxide, ethylene and others). Previous studies have indicated that the activity of protopanaxadiol 6-hydroxylase ( $\mathrm{P} 6 \mathrm{H})$, one of the enzymes involved in the ginsenoside pathway, is dependent on molecular oxygen and carbon monoxide (Yue et al. 2008). This enzyme converts the ginsenoside aglycone protopanaxadiol into protopanaxatriol, and so increased $\mathrm{P} 6 \mathrm{H}$ activity may result in increased protopanaxatriol-type ginsenoside content. In the present study, significantly higher levels of protopanaxatriol derivatives, such as metabolites $\operatorname{Rg} 1$ and Re, were observed in bioreactor cultures than the flask cultures, suggesting that the conditions in the bioreactor were more favorable for the activation of $\mathrm{P} 6 \mathrm{H}$ than shake flasks. Our findings also indicate that greater amounts of protopanaxadiol derivatives were produced in transformed $P$. quinquefolium roots at scale-up conditions. These results suggest that the bioreactor root cultures demonstrate higher glucosyltransferase activity, which is needed to create individual 


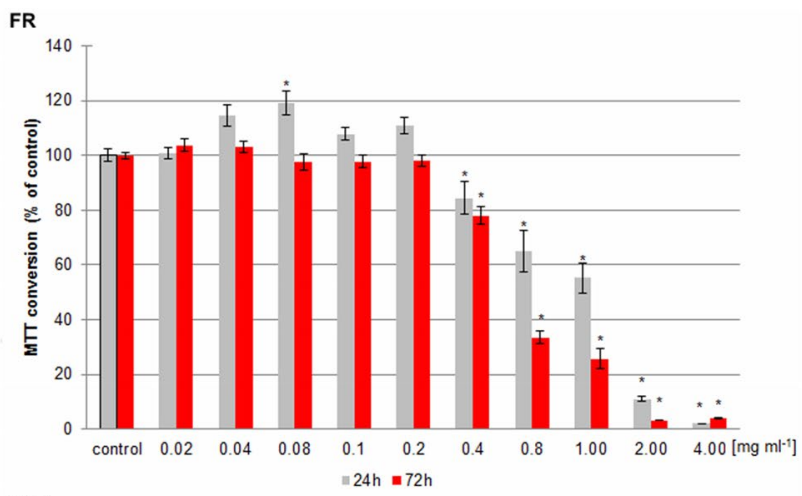

FHR

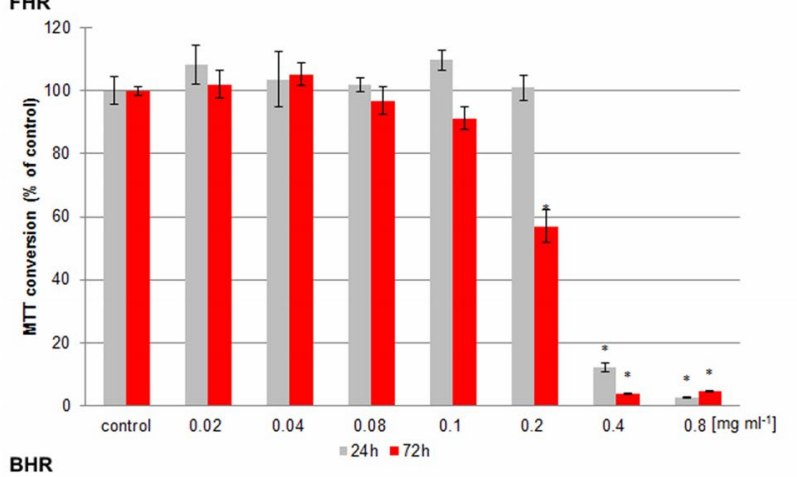

BHR

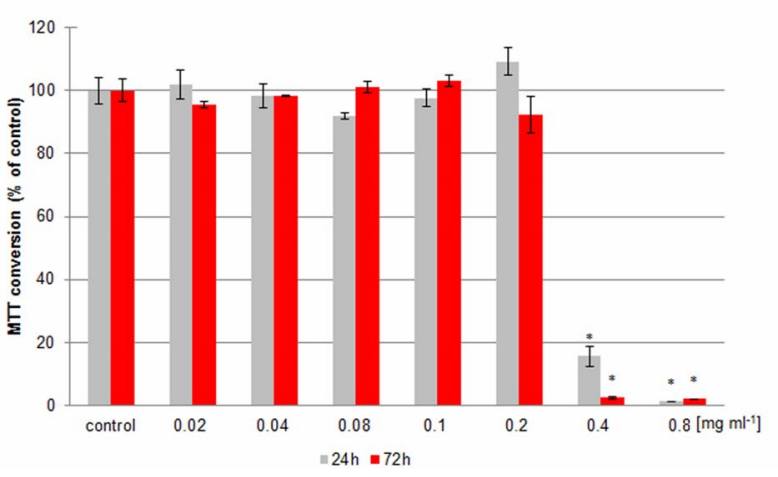

Fig. 1 The effects of the extracts of hairy root culture (cultivated in bioreactor (BHR) and shake flasks (FHR)) and roots of field (FR) grown $P$. quinquefolium plants on the viability of the glioblastoma cell line (T98G). Bars represent the mean \pm SE. $* p<0.05$ versus control

metabolites in the final step of the ginsenoside pathway. However, these enzymes and the factors regulating their activity remain still poorly understood. On the other hand Lu et al. (2017) confimed that UDP-glucosyltransferate gene activity can be regulated and its overexpression can led to increased accumulation of $P q 3-O-U G T 1$ mRNA and higher level of ginsenosides belonging to $\mathrm{Rb}$ group.

This present report is the first to identify and quantitatively analyze the phenolic compounds present in $P$. quinquefolium hairy roots. Our findings show that the content of identified phenolic compounds differed significantly between samples. The main phenolic compound in hairy roots of $P$. quinquefolium cultivated both in small scale culture and scale-up was chlorogenic acid. Meanwhile in the $P$. ginseng adventitious root extract, ferulic acid was found to predominate $\left(90 \mu \mathrm{g} \mathrm{g}^{-1} \mathrm{~d}\right.$.w.) (Sivakumar and Paek 2005). This compound was also identified in our samples (BHR, FR), but at a lower level.

The total phenolic compound level (TPC; determined using Folin-Ciocalteu method) of the $P$. quinquefolium hairy roots was found to be similar with that of a previously tested $P$. ginseng hairy root culture (Jeong et al. 2007). Moreover, our shake flasks and bioreactor cultures demonstrated significantly higher TPC levels than field-grown American ginseng root and hydroponic-cultured Asian ginseng roots (Hwang et al. 2014).

\section{Antioxidant activity}

The transformed root had the ability to reduce metal irons and scavenge free radicals; however, the antioxidant potential of the analysed extracts was lower than those of more well-known antioxidant products, such as blueberries or blackberries (respectively, 257 and $344 \mu \mathrm{mol} \mathrm{Fe(II)} \mathrm{g}^{-1}$ d.w. of extract) (Henríquez et al. 2011) or cranberry powder (242-319 $\mu$ mol Fe(II) $\mathrm{g}^{-1}$ d.w.) (Kim et al. 2014a, b). On the other hand, the antioxidant properties of American ginseng extracts were stronger than those of other medicinal plants such as Aloe vera $\left(37 \mu \mathrm{mol} \mathrm{Fe}(\mathrm{II}) \mathrm{g}^{-1}\right.$ d.w.) or Coriandrum

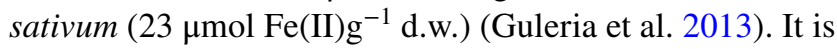
not surprising that $P$. quinquefolium hairy roots (HR) have antioxidant potential, as they are known to contain phenolic compounds. Chlorogenic acid, the main phenolic compound identified in HR extracts, is a known antioxidant (Liang and Kitts 2016; Grzegorczyk-Karolak et al. 2015). In addition, the relationship between antioxidant potential and the level of phenolic compounds is well documented (Guleria et al. 2013; Henríquez et al. 2011; Laguerre et al. 2011). Such a relationship was also noticed in the current study; stronger antioxidant effects were observed for HR extracts, containing higher total phenol levels than the FR extracts. On the other hand, it was observed that the BHR extract, characterized by high TPC and TGC values, demonstrated greater antioxidant activity than the FHR extract, which possesses higher phenol content but is deficient in ginsenosides. These findings suggest that the antioxidant activity of $P$. quinquefolium hairy root extracts can be attributed to the additive or synergic effect of phenolic compounds and various ginsenosides. Also, the antioxidant capacities of leaf extracts from traditionally-cultivated ginseng plants has been found to be related to their ginsenoside content (Zhang et al. 2014).

Additionally, our results suggest that the antioxidant effect of the tested extracts may be particularly associated with the content of some ginsenosides, such as $\mathrm{Rb} 2$, $\mathrm{Rd}$ and $\operatorname{Rg} 1$, whose levels were found to be higher in the in vitro cultures than the FR extract. This is confirmed by 
previous studies indicating that the individual ginsenoside (20(S)-protopanaxadiol or 20(S)protopanaxatriol) displays stronger antioxidant activity when a glucose is attached to the 20-position of the triterpene dammarane, such as in the $\mathrm{Rd}$ and $\mathrm{Rg} 1$ molecules (Lü et al. 2009). Additionally Chae et al. (2010) indicate that the hetero-sugar (glucosearabinose) linkage, present in $\mathrm{Rb} 2$ or $\mathrm{Rc}$, showed stronger antioxidant activity than metabolite Rb1, which is a homodisaccharide glycoside. The antioxidant ability of Rd has also been confirmed in vivo, where it enhanced the activities of antioxidant enzymes, including superoxide dismutase, catalase and glutathione peroxidase (Zhang et al. 2013).

\section{The effects of FHR and BHR on the viability of glioblastoma cell line (T98G)}

Another novel aspect of the present study is that it examines the cytotoxicity potential of the $P$. quinquefolium hairy root extracts, more specifically, against a human glioblastoma cell line (T98G). All tested extracts showed a cytotoxic effect against the T98G line, but with varying potency. An analysis of $\mathrm{IC}_{50}$ values indicated that the two extracts from in vitro culture (FHR, BHR) showed very similar cytotoxic potency, and that this was about four-fold stronger than for the FR extract.

Extracts from traditionally-cultivated Panax ginseng plants have been found to have anti-carcinogenic effects against various human cancer cell lines (stomach, cervical, colorectal, breast adenocarcinoma, lung carcinoma) (Lee et al. 2014, 2016) with $\mathrm{IC}_{50}$ values ranging between 0.25 and $2.84 \mathrm{mg} \mathrm{mL}^{-1}$ depending on the tested cell line (Lee et al. 2014, 2016); similar values were obtained in the present study for our $P$. quinquefolium extracts. In contrast, Park et al. (2016) reported $P$. ginseng root extract to have much stronger anticancer activity against prostate cancer cell lines $\left(\mathrm{IC}_{50} 0.05-0.075 \mathrm{mg} \mathrm{mL}^{-1}\right.$ ).

Previous studies have found single saponins to have antiproliferation activity, for example $\mathrm{Rg} 1$ inhibited colon cancer growth, and $\mathrm{Rb} 2$ and $\mathrm{Rg} 1$ were effective against lung cancer cells (Lee et al. 2016; Chen et al. 2016). Higher levels of these saponins were produced in the HR extracts than in field-cultivated plant material, which may account for the much higher cytotoxic activity observed for the HR than the FR extract. Our findings also suggest that phenolic compounds may also play a role: despite being poor in ginsenosides, the FHR extract demonstrated similar anti-cancer potential to the BHR extract. One possible explanation could be the high level of chlorogenic acid level found in the FHR cultures, as chlorogenic acid has been found to inhibit tumor growth and angiogenesis in several types of malignancy, including glioblastoma (Xue et al. 2017).

\section{Conclusions}

The present study represents the first qualitative and quantitative determination of phenolic compounds in hairy root cultures of $P$. quinquefolium. The LC-MS/MS analysis revealed the presence of 10 phenolic acids and six flavonoids, of which chlorogenic acid predominated in all in vitro material.

The bioreactor-cultured transformed roots showed the highest antioxidant activity, as well as high production of phenols and ginsenosides, suggesting that ginsenosides and phenolic compounds may play an important role as antioxidants. The extracts from the in vitro cultures also demonstrated stronger cytotoxic properties than those from the roots of the field-grown plants. However, further detailed in vivo studies are necessary to evaluate the biological potential of American ginseng hairy root cultures and their possible value in the pharmaceutical or food industry.

Author contributions EK designed the study, obtained and maintained hairy root cultures, prepared the extracts for study, and wrote the paper. GS determined the ginsenoside content by HPLC. MW-made LCMS/MS and HPLC analysis of phenolic compounds, AW-O and MJ-B determined the cytotoxic properties of the hairy root extract toward T98G line. IG-K provided substantive consultations and took part in describing the manuscript. All authors read and approved the final manuscript.

Funding The research reported in this manuscript was supported by Grants Nos. 50213771 and 503/3-012-02/503-31-001 from the Medical University of Łodz.

\section{Compliance with ethical standards}

Conflict of interest The authors declare that they have no conflict of interest.

Open Access This article is distributed under the terms of the Creative Commons Attribution 4.0 International License (http://creativeco mmons.org/licenses/by/4.0/), which permits unrestricted use, distribution, and reproduction in any medium, provided you give appropriate credit to the original author(s) and the source, provide a link to the Creative Commons license, and indicate if changes were made.

\section{References}

Bathoju G, Rao K, Giri A (2017) Production of sapogenins (stigmasterol and hecogenin) from genetically transformed hairy root cultures of Chlorophytum borivilianum (Safed musli). Plant Cell, Tissue Organ Cult 131:369-376

Biswas T, Mathur AK, Mathur A (2017) A literature update elucidacing production of Panax ginsenosides with a special focus on strategies enriching the anti-neopladstic minor ginsenosides in ginseng preparation. Appl Microbial Biotechnol 101(10):4009-4032 
Brown PN, Yu R, Cain T, Huite G, Jin CD, Kababick JN, Leong G, LeVanseler K, Lunetta S, Ma YC, Reif K, Schaneberg B, Shevchuk C, Smith R, Sullivan D, Wijewickreme N, Windust A (2013) Determination of ginsenoside content in Panax ginseng C.A. Meyer and Panax quinquefolius L. root materials and finished products by high-performance liquid chromatography with ultraviolet absorbance detection: interlaboratory study. J AOAC Int 96(1):12-19

Chae S, Kang KA, Youn O, Park JS, Hyun JW (2010) A comparative study of the potential antioxidant activities of ginsenosides. J Food Biochem 34:31-34

Chen XJ, Zhang XJ, Shui YM, Wan JB, Gao JL (2016) Anticancer activities of protopanaxadiol- and protopanaxatriol-type ginsenosides and their metabolites. Evid Based Complement Altern Med. https://doi.org/10.1155/2016/5738694

Christensen LP (2009) Ginsenoside chemistry, biosynthesis, analysis and potential health effects. Adv Food Nutr Res 55:1-99

Grzegorczyk-Karolak I, Kuźma Ł, Wysokińska H (2015) Study on the chemical composition and antioxidant activity of extracts from shoot culture and regenerated plants of Scutellaria altissima L. Acta Physiol Plant 37(1):1736

Guleria S, Tiku AK, Singh G, Koul A, Gupta S, Rana S (2013) In vitro antioxidant activity and phenolic contents in methanolic extractsfrom medicinal plants. J Plant Biochem Biotechnol 22:9-15

Ha LT, Pawlicki-Jullian N, Pillon-Lequart M, Boitel-Conti M, Duong HY (2016) Gontier E (2016) Hairy root cultures of Panax vietnamensis, a promising approach for production of ocotillol-type ginsenosides. Plant Cell, Tissue Organ Cult 126:93-103

Henríquez C, López-Alarcón C, Gómez M, Lutz M, Speisky H (2011) Time-dependence of ferric reducing antioxidant power (FRAP) index in Chilean apples and berries. Arch Latinoam Nutr 61:323-332

Hwang CR, Lee SH, Jang GY, Hwang IG, Kim HY, Woo KS, Lee J, Jeong HS (2014) Changes in ginsenoside compositions and antioxidant activities of hydroponic-cultured ginseng roots and leaves with heating temperature. J Ginseng Res 38:180-186

Inomata S, Yokoyama M, Gozu Y, Shimizu T, Yanagi M (1993) Growth pattern and ginsenoside production of Agrobacteriumtransformed Panax ginseng roots. Plant Cell Rep 12:681-686

Jang D, Lee J, Eom SH, Lee SM, Gil J, Lim HB, Hyun TK (2016) Composition, antioxidant and antimicrobial activities of Eleutherococcus senticosus fruit extracts. J Appl Pharm Sci 6(3):125-130

Jeong GT, Woo JCh, Park DH (2007) Effect of plant growth regulators on growth and biosynthesis of phenolic compounds in genetically transformed hairy roots of Panax ginseng C. A. Meyer. Biotechnol Bioprocess Eng 12(2):86-91

Kang OJ, Kim JS (2016) Comparison of ginsenoside contents in different parts of Korean Ginseng (Panax ginseng C.A. Meyer). Prev Nutr Food Sci 21(4):389-392

Kasote DM, Katyare SS, Hegde MV, Bae H (2015) Significance of antioxidant potential of plants and its relevance to therapeutic applications. Int J Biol Sci 11(8):982-991

Kim MJ, Kim JH, Kwak HK (2014a) Antioxidant effects of cranberry powder in lipopolysaccharide treated hypercholesterolemic rats. Prev Nutr Food Sci 19:75-81

Kim YJ, Jeon JN, Jang MG, Oh JY, Kwon WS, Jung SK, Yang DCH (2014b) Ginsenoside profiles and related gene expression during foliation in Panax ginseng Meyer. J Ginseng Res 38(1):66-72

Kochan E, Królicka A, Chmiel A (2012) Growth and ginsenoside production in Panax quinquefolium hairy roots cultivated in flasks and nutrient sprinkle bioreactor. Acta Physiol Plant 34:1513-1518

Kochan E, Szymańska G, Szymczyk P (2014) Effect of sugar concentration on ginsenoside biosynthesis in hairy root cultures of Panax quinquefolium cultivated in shake flasks and nutrient sprinkle bioreactor. Acta Physiol Plant 36(3):613-619
Kochan E, Szymanska G, Grzegorczyk-Karolak I (2015) The extracts from Panax quinquefolium shoots derived from somatic embryos accumulate ginsenosides and have the antioxidant properties. Vitro Cell Dev Biol Plant 51(6):696-701

Kochan E, Kuźma Ł, Szymczyk P, Szymańska G (2016) Nitrogen and phosphorus as the factors affecting ginsenoside production in hairy root cultures of Panax quinquefolium cultivated in shake flasks and nutrient sprinkle bioreactor. Acta Physiol Plant 38:149

Kochan E, Szymczyk P, Kuźma Ł, Lipert A, Szymańska G (2017) Yeast extract stimulates ginsenoside production in hairy root cultures of American ginseng cultivated in shake flasks and nutrient sprinkle bioreactor. Molecules 22(6):880

Laguerre M, Chen B, Lecomte J, Villeneuve P, McClements DJ, Decker EA (2011) Antioxidant properties of chlorogenic acid and its alkyl esters in stripped corn oil in combination with phospholipids and/ or water. J Agric Food Chem 59(18):10361-10366

Lee ChH, Kim JH (2014) A review on the medicinal potentials of ginseng and ginsenosides on cardiovascular diseases. J Ginseng Res 38:161-166

Lee KA, Kim KT, Chang PS, Paik HD (2014) In vitro cytotoxic activity of ginseng leaf/stem extracts obtained by subcritical water extraction. J Ginseng Res 38:289-292

Lee DG, Jang SI, Kim YR, Yang KE, Yoo SJ, Lee ZW, An HJ, Jang IS, Choi JS, Yoo HS (2016) Anti-proliferative effects of ginsenosides extracted from mountain ginseng on lung cancer. Chin J Integr Med 22(5):344-352

Liang N, Kitts DD (2016) Role of chlorogenic acids in controlling oxidative and inflammatory stress conditions. Nutrients 8(1):16

Linh NTN, Cuong LK, Tam HT, Tung HT, Luan VQ, Hien VT, Loc NH (2019) Nhut DT (2019) Improvement of bioactive saponin accumulation in adventitious root cultures of Panax vietnamensis via culture periods and elicitation. Plant Cell, Tissue Organ Cult 137:101-113

Lu C, Zhao SJ, Wang XS (2017) Functional regulation of a UDPglucosyltransferase gene (Pq3-O-UGT1) by RNA interference and overexpression in Panax quinquefolius. Plant Cell, Tissue Organ Cult 129:445-456

Lü JM, Yao Q, Chen Ch (2009) Ginseng compounds: an update on their molecular mechanisms and medical applications. Curr Vasc Pharmacol 7(3):293-302

Mallol A, Cusidó RM, Palazón J, Bonfill, M, Morales C, Piñol MT (2001) Ginsenoside production in different phenotypes of Panax ginseng transformed roots. Phytochemistry 57(3):365-371

Marrelli M, Conforti F, Araniti F, Statti GA (2016) Effects of saponins on lipid metabolism: a review of potential health benefits in the treatment of obesity. Molecules 21:1404

Matkowski A (2008) Plant in vitro cultures for production of antioxidants-a review. Biotechnol Adv 26:548-560

Park JY, Choi P, Kim K, Kang KS, Ham J (2016) Increase in apoptopic effect of Panax ginseng by microwave processing in human prostate cancer cells: in vitro and in vivo studies. J Ginseng Res 40:62-67

Pawar VS, Shivakumar H (2012) A current status of adaptogens: natural remedy to stress. Asian Pac J Trop Dis 2(suppl 1):S480-S490

Radad K, Moldzio R, Rausch W-D (2011) Ginsenosides and their CNS targets. CNS Neurosci Ther 17:761-768

Shi W, Wang Y, Li J, Zhang H, Ding L (2007) Investigation of ginsenosides in different parts and ages of Panax ginseng. Food Chem 102(3):664-668

Sivakumar G, Paek KY (2005) Methyl jasmonate induce enhanced production of soluble biophenols in Panax ginseng adventitious roots from commercial scale bioreactors. Chem Nat Compd 41(6):669-673

Sukweenadhi J, Kim YJ, Rahimi S, Silva J, Myagmarjav D, Kwon WS, Yang DC (2017) Overexpression of a cytosolic ascorbate 
peroxidase from Panax ginseng enhanced salt tolerance in Arabidopsis thaliana. Plant Cell, Tissue Organ Cult 129:337-350

Sun S, Qi LW, Du GJ, Mehendale SR, Wang ChZ, Yuan ChS (2011) Red notoginseng: higher ginsenoside content and stronger anticancer potential than Asian and American ginseng. Food Chem 125(4):1299-1305

Tung NH, Uto T, Morinaga O, Kim YH, Shoyama Y (2012) Pharmacological effects of ginseng on liver functions and diseases: a minireview. Evid Based Complement Altern Med 173297:1-7

Van Breusegem F, Dat JF (2006) Reactive oxygen species in plant cell death. Plant Physiol 141(2):384-390

Wang Z, Zhang H (2012) Antidiabetic effects of ginseng in humans and rodents. J Metab Synd 1(2):106

Wang J, Gao W, Zuo B, Liu H, Zhang L, Huang L (2012) Gradually scale-up culture in a bioreactor promotes radical scavenging activity of Panax ginseng (C. A. Meyer) adventitious roots on 1,1-diphenyl-2-picrylhydrazyl. Plant Growth Regul 67(1):101-105

Wang ChZ, Cai Y, Anderson S, Yuan CS (2015) Ginseng metabolites on cancer chemoprevention: an angiogenesis link? Diseases 3:193-204
Xue N, Zhou Q, Ji M, Jin J, Lai F, Chen J, Zhang M, Jia J, Yang H, Zhang J, Li W, Jiang J, Chen X (2017) Chlorogenic acid inhibits glioblastoma growth through repolarizating macrophage from M2 to M1 phenotype. Sci Rep 7(39011):1-11

Yue CJ, Zhou X, Zhong JJ (2008) Protopanaxadiol 6-hydroxylase and its role in regulating the ginsenoside heterogeneity in Panax notoginseng cells. Biotechnol Bioeng 1:933-940

Zhang YX, Wang L, Xiao EL, Li SJ, Chen JJ, Gao B, Min GN, Wang ZP, Wu YJ (2013) Ginsenoside-Rd exhibits anti-inflammatory activities through elevation of antioxidant enzyme activities and inhibition of JNK and ERK activation in vivo. Int Immunopharmacol 17(4):1094-1100

Zhang YC, Li G, Jiang C, Yang B, Yang HJ, Xu HY, Huang LQ (2014) Tissue-specific distribution of ginsenosides in different aged ginsengand antioxidant activity of ginseng leaf. Molecules 19:17381-17399

Publisher's Note Springer Nature remains neutral with regard to jurisdictional claims in published maps and institutional affiliations.

\section{Affiliations}

\section{Ewa Kochan ${ }^{1}$ - Grażyna Szymańska ${ }^{1}$ (1) Marzena Wielanek ${ }^{2}$ - $\cdot$ Anna Wiktorowska-Owczarek ${ }^{3}$. Marta Jóźwiak-Bębenista ${ }^{3}$ (1) Izabela Grzegorczyk-Karolak ${ }^{4}$ (1)}

Grażyna Szymańska

grazyna.szymanska@umed.lodz.pl

Marzena Wielanek

marzena.wielanek@biol.uni.lodz.pl

Anna Wiktorowska-Owczarek

anna.wiktorowska-owczarek@umed.lodz.pl

Marta Jóźwiak-Bębenista

marta.jozwiak-bebenista@umed.lodz.pl

Izabela Grzegorczyk-Karolak

izabela.grzegorczyk@umed.lodz.pl
Department of Pharmaceutical Biotechnology, Medical University of Lodz, Muszyńskiego 1, 90-151 Lodz, Poland

2 Department of Plant Physiology and Biochemistry, University of Lodz, Banacha 16, Lodz, Poland

3 Department of Pharmacology and Toxicology, The Interfaculty Chair of Basic and Clinical Pharmacology and Toxicology, Medical University of Lodz, Żeligowskiego, 90-151 Lodz, Poland

4 Department of Biology and Pharmaceutical Botany, Medical University of Lodz, Muszyńskiego 1, 90-151 Lodz, Poland 